\title{
Virtual Reality Based End-User Assessment Tool for Remote Product and System Testing
}

\author{
Burcu Dolunay ${ }^{1}$ \\ b_doluna@alcor.concordia.ca \\ Department of Mechanical and Industrial Engineering \\ Ali Akgunduz ${ }^{1}$ \\ akgunduz@encs.concordia.ca \\ ${ }^{1}$ Concordia University \\ 1455 de Maisonneuve Blvd. West \\ Montreal, Quebec, Canada
}

\begin{abstract}
It is known that a through understanding of the end-user is the most valuable information to improve design, training, maintenance and assembly/disassembly processes of products and systems. The most widely used method to analyze human-system interaction, user experiments, which requires a product prototype, a test environment and a researcher to watch and collect data. This results in a procedure that is time consuming and limited by geographical constraints. This paper proposes a virtual reality based automated user assessment tool. An event based data collection and analysis approach is designed. Initial results support the usefulness of method as an automated tool to detect problems in an evaluated system
\end{abstract}

\section{Introduction}

Today designers and researchers are continuously seeking optimal product design solutions that lead to more user friendly products which are safer to use, easier to maintain, and easier to assembly and disassembly. Designing such products is only possible by the collaborative work of both designers and the end-users of the products (customers). Through usability tests on the actual prototypes under the supervisions of the testers (observers), crucial user data can be collected for further analysis. In traditional test environments users perform certain given tasks with the system. Consequently, testers observe the collected data to detect problems in the product/system.

In remote usability tests the subjects use virtual replicas of the examined systems. Availability of virtual prototypes and remote tests fulfill a big need in the sense that they enable to evaluate products or systems in a geographically wide area where products may have several different potential user groups, or companies have product development in several places. However, many remote testing settings record and transmit video and audio data to be analyzed afterwards (Kuutti et al., 2001, Hammontree et al., 1994). This means although the test is conducted in a computer environment the data analysis still requires an expert interaction which is time consuming and prevents the application of a test to a larger number of subjects. Moreover, observational data analysis performed without software support provides qualitative results recorded by a human observer during the experiment. On the other hand if data is captured and analyzed electronically, investigators will also have the numerical results obtained from analyses as well (Sanderson et al., 1994). Such kind of integrated analysis may reveal behavioral patterns that designers are not aware off. But the problem with automated data collection and the analysis of humansystem interaction summary (data stored in log files) is that since a log record system can not work as a filter like a human observer does; there is generally a huge amount of data for analysis to extract useful information. Hence an automated data collection system that records the user's interactions with the proposed product design into log files and a smart analysis tool to examine these files in order to derive meaningful insights about the usability, maintainability and ease-of-assembly of the product is beneficial in product evaluation.

The main objective of this study is to propose a virtual reality based information collection and analysis method to understand user behavior over the designed systems and suggest appropriate design 
changes to better comply with the various user groups so the final design is easy to use, maintain or assemble. To achieve the proposed goals, current virtual reality technology and simple database management techniques are used. An event based approach is proposed to summarize human-system interaction. The emphasis is given to the intelligent user behavior analysis approach.

\section{Literature Review}

The methodology, proposed in this paper, has two important aspects, first developing a virtual reality based automated data collection scheme; and second developing an automated data analysis technique to mine useful information in data. VR has already been successfully applied to simulation of many complex systems to collect data. On the other hand, when it comes to collecting and analyzing behavioral data from virtual reality systems, there are not many general-purpose studies. Automation of data analysis has been widely studied and applied so far by humancomputer interaction researchers in evaluating user interfaces. An extensive review of literature for software evaluation tools can be found in Hilbert et al. (2000) and Ivory et. al (2000). Here will be reviewed some of the studies that use one of the methods closer to the ones used in this research work like metric based analysis or pattern comparison. Metric-based analysis generates quantitative results from the data genarally by statistical methods like reporting average performance time, error frequency etc. Sanderson et al. (1994) is an example to the tools that provides this kind of statistical information. It also provides a pattern matching functionality by aligning the actual logs with the process model and displaying the differences in a spreadsheet format. Paternò and Lecerof (1998) also includes pattern comparison where user logs are compared with the previously prepared process model to detect violations of prerequisites, to see completed or failed tasks and errors.

Having one or two common ideas with each of these pattern comparison based studies in our approach we do not take the logs as they are but rather extract paths in logs of users. One approach to path extraction, is a version of sequential patterns' mining problem in data mining. Discovering patterns in a sequence of events has been studied extensively in the literature for different purposes. In their works, Agrawal and Srikant (1995) propose Apriori Algorithms, where they mine sequential purchase patterns for customers in a large database of purchased items. In their problem definition the purchases need not be contiguous to form patterns thus is not very applicable to traversal pattern mining however, there are numerous methodologies based on this study for contiguous pattern mining like methods developed to mine web usage data. In their work Chen et al. (1998) proposed an algorithm to convert the original sequence of $\log$ data into a set of "maximal forward references" which implies the longest path ever followed by the user without any interruption or cancellation in paths. In another study, Dunham and Xiao (2001) proposed an algorithm to find traversal patterns in web usage logs this time keeping repetitions and backward moves in paths.

Another approach is to extract user behavior from usage data is deducing a grammar from log files. For grammar deduction, there have been studies that propose different methods depending on the complexity and characteristics of the system. Firstorder Markov chains are used to construct probabilistic models for web navigation sessions called Hypertext Probabilistic Automaton (HPA) (Borges, 2000). Cook (1996) discovers software process models from execution logs and then validates the model by quantitatively measuring the level of correspondence between the model and the logs. Agrawal et al. (1997), presents a methodology to construct process flow diagrams from unstructured past executions.

Although, there are studies in web-mining towards the understanding of users particularly in webshopping, not many studies in the literature that discuss automated analysis of user-system interaction in virtual reality simulations. The main challenge in design of such automated assessment tool is that a virtual reality simulation is capable of recording a vast amount of data without any filtering. To overcome this problem, in this research work we designed a VR based user assessment simulator that captures usersystem interaction in the forms of events. Finally a data-mining technique is introduced to identify differences and similarities between users during the use of the system. Considering the wide range of different studies that are related to different parts of the methodology presented in this work, the approach presented offers distinct advantages in terms of starting point, use of hidden knowledge in the logs and the application area.

\section{Model: Four Step Data-Mining Process}

In this work we are concerned with the understanding of user behavior during their interaction with a system (product). We are specifically interested in discovering patterns followed by users and getting 
most out of the usability data both in qualitative and quantitative means. Hence the objective is to develop a VR based automated end-user assessment tool that can achieve above functionalities without massive human effort. The proposed method draws on two main efforts for this purpose:

i. constructing virtual simulations (e.g. a prototype, an assembly or a maintenance scenario) and capturing interactions of the user with the virtual prototype of product/system in the form of transcripts

ii.developing an intelligent and automated data analysis technique to get most out of the voluminous data.

Today's VR equipment is varied in functionality and purpose and in most of the applications the actions of the subject can be readily captured at the desired level of detail. The greater challenge is in controlling, analyzing and deriving meaningful conclusions from the available vast amount of data. In the proposed work, our main objective is to propose better methods to analyze the data so that product/system designers can better address the needs and requirements of the end-users. A good way of understanding end-users behaviors or identifying problematic parts in a system is to know the paths that users follow to perform certain tasks, or the paths that enable highest level of effectiveness in terms of time and energy usage. Furthermore identifying the characteristics of users who follow similar patterns during the task provide important insight to the designers. In an attempt to answer these questions we suggest a four-phase analysis approach: i) Recording user interaction with the system as a set of events in the user log files; ii) Scanning log files for potential indicators of problems and obtaining performance parameters for users; iii) Clustering users with respect to the observed results; iv) Extracting paths in each cluster and comparing these paths with the designer data and/or among user clusters.

Each of the above steps has been used previously in the literature to understand different systems. Our attempt in this research work is to use the results of each phase to feed the consecutive phase and then integrate the results at the end in an attempt to analyze user-system interaction during a virtual reality simulation. To the best of our knowledge, the integration of all four phases has not been addressed in the literature to assess user-environment interaction in a virtual reality simulation.

Phase 1: Recording user interaction with the system as a set of events.
In the first phase, all user input and system output are recorded as a set of events where events are welldefined, instantaneous actions that characterize a change in the system. Also desired statistics about the session are also recorded here, like session duration. At the end of each user session, a $\log$ file $R_{i}$ is obtained in the form of:

$$
R_{i}=\left\langle(i),\left(e_{1}, e_{2} \ldots \ldots . e_{n}\right),\left(a_{1}, a_{2} \ldots \ldots \ldots a_{m}\right)\right\rangle
$$

where

$$
\begin{aligned}
& i=\text { Identification number of a user } \\
& E_{i}:\left(e_{1}, e_{2}, \cdots, e_{m}\right)_{i}: \text { Event stream of user } \mathrm{i} \\
& A_{i}=\left(a_{1}, a_{2}, \cdots a_{m}\right)_{i} \text { : Set of recorded statistics }
\end{aligned}
$$

for the session of user $\mathrm{i}$

$$
\begin{aligned}
a_{j} & =\text { Record } j \\
e_{j} & =j^{t h} \text { event in the log file } \\
\mathbf{R} & =\left[\begin{array}{llll}
R_{1} & R_{2} & \cdots & R_{n}
\end{array}\right]
\end{aligned}
$$

Phase 2: Calculating 'Performance Parameters' using Potential Indicators

One of the key benefits of collecting the interaction data with its all details is that we can collect all the relevant numeric data which is closely related to the usability of the product. In human-computer interfaces literature there are many metrics reported to be useful to catch more insight about the performance of the user with an interface such as delays, ruptures, repetitions, performance time etc (Hilbert et al., 2000). For any product or system, the designers can collaboratively decide on potential indicators that may apply best to their situation. Then for each subject, these predetermined metrics are calculated from the data collected from user's log. General statistics packages or custom calculation methods can be preferred depending on need.

Phase 3: Clustering users with respect to the results

Once the performance parameters are obtained, the next step is clustering the large sample space into several classes based on performance indicators of individuals. Clustering is one of the most studied topics in data mining literature. The aim of clustering in this work is to come up with the clusters that represent the sample space in terms of performance indicators. In another word, the objective is collecting users with similar results in the same cluster. Different methods can be adopted here for different cases depending on the needs, namely the number and kind of included dimensions, number of subjects, etc. Comparison of emerging paths from different clusters 
provides insight about the relative performance of the users of a certain paths. Hence, the designer sees differences between paths of low and high performance users. So if clustering process results with $k$ clusters, we have $k$ files containing logs of its member subjects as shown in figure 1. In the figure, event streams of subjects (set $E$ ) in the same cluster are collected in separate files and event stream of a user is the set that contains sequence of events executed by that user.

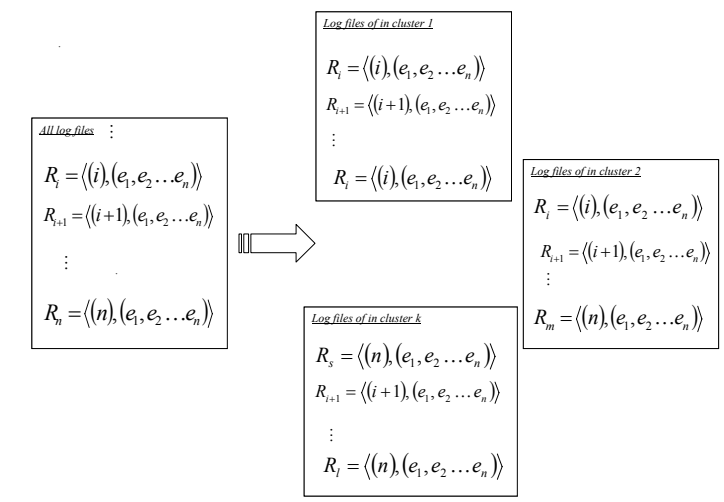

Figure 1: Output of clustering phase

Phase 4: Extracting followed paths in each cluster and comparing these paths with the designer data and/or among user clusters.

Once the $\log$ files of users are clustered, the next phase is to filter the logs or to extract meaningful paths from the log files that are unnecessarily large and can be misleading due to noise in the data. This noise can be interruptions in the path followed by repetitions, cancellations or some basic actions that are not necessary in the analysis. Moreover, comparison without filtering may lead to erroneous results. Oneto-one comparison of user-logs gives no common paths since it is improbable for logs of two people are completely the same even though they have followed similar patterns. The problem here is to find recurring traversal patterns in the logs in each cluster.

First approach is to cast the problem as one of mining traversal patterns in noisy data and find the frequent paths of each cluster. This problem has been studied extensively in the data mining literature for different purposes and numerous methodologies have been proposed, most of which are Apriori based approaches. They function in two steps: first finding the paths in each user-log, then scanning the path database and choose the paths that were repeated at least by the required number of times. The final result for this approach is a set of preferred paths as illustrated in figure 2 .

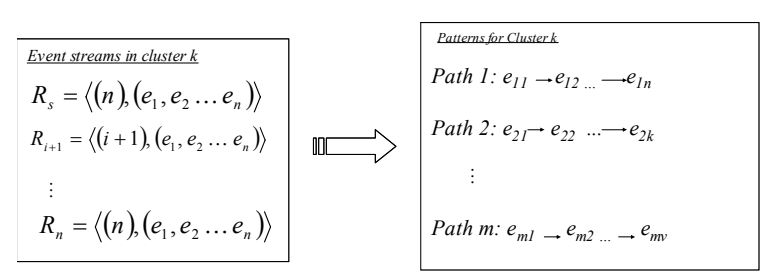

Figure 2 : Set of the preferred paths for cluster $k$ mined from the set of event streams

Another approach is to build a process model from logs or in other words; to deduce a grammar for the user session from sets of execution data. This method results with a model representing the process that is embedded in the logs as in figure 3.

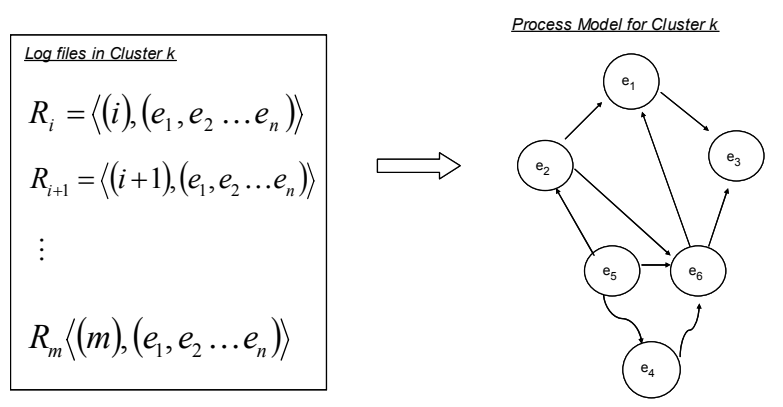

Figure 3: Process model for the cluster $k$

There is a wide range of methods for grammar inference from a data set. Some of the well-known methods are; algorithms that make use of prefix trees to iteratively build the models; statistical inference techniques that make use of Hidden Markov Models and Neural Nets that use neural networks techniques (Cook, 1996).

The approach chosen to extract paths at this phase is depend on the characteristics of the system/product in question together with the definition of a "pattern" for the system meaning what information we want to include or exclude in forming the patterns. The researchers decide what is required in their system to form a pattern, what kind of events they want to include in the paths or what kind of comparison is planned to be used in the end. In our experiment we use maximal forward sequences algorithm (Chen et al., 1998) to extract paths in an event stream. After extracting paths for each log in a cluster, the subset of paths repeated by a certain number were chosen from the entire set. In this path elimination process, we introduced a "similarity coefficient for the paths" to obtain the paths that are not $100 \%$ matching each other 
but satisfy a certain proportion of the common characters.

\section{Experiments: Virtual Assembly simulation}

To support the proposed methodology in this paper, a process simulation was designed using the Visual $\mathrm{C}++$ and Inventor library. Two individual experiments were designed. In the first experiment, we asked 16 subjects to complete an assembly process of a product in our virtual reality simulation (figure 4-a: components of a wall-mounted bookshelf). We collected and analyzed the data from this first group to see if our analysis approach can detect the problematic parts in the system. In the second test, we improved those problematic parts that are captured in the first phase. This time, a total of 12 subjects are asked to complete the assembly in the modified version of the virtual reality simulator (figure 4-b: different screws are differentiated by coloring).
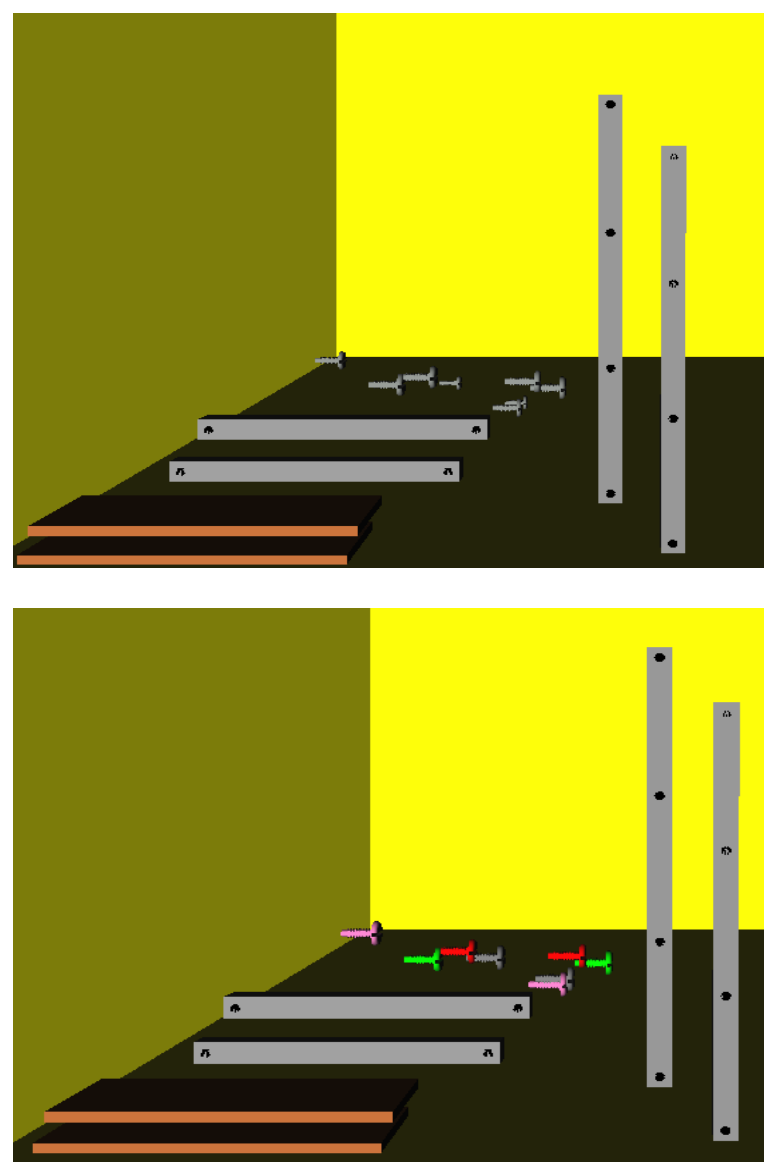

Figure 4: a: Assembly scenario includes obvious problem for the assembler. b: Improved assembly scenario
The objective of the second part was to see if we could improve the results with the information obtained from the analysis of first experiment. In our experiment we determined number of repetitions $\left(\mathrm{K}_{\mathrm{i}}\right)$, cancellations $\left(\mathrm{C}_{\mathrm{i}}\right)$ and task completion time $\left(\mathrm{T}_{\mathrm{i}}\right)$ as performance indicators and calculated these values for each subject as described in phase 2 . In the third phase, we used kmeans clustering algorithm on multiple dimensions to classify the subjects based on their results. In the fourth phase, for the pattern extraction we used the maximal forward sequences algorithm from Chen et al. (1998). In order to find frequent paths we devised a scanning algorithm to eliminate paths that repeated less than a certain number of times. Also we devised a sequence comparison algorithm to detect deviations from process model and applied to the data. Our results show that the statistical and paths analysis detect the problem of the system when interpreted together. Also in addition to problematic points, the analysis revealed a behavioral pattern that was unknown to experimenters before the experiment. This result was also supported by direct observation of test subjects by an instructor during the experiment.

As a result of our analysis technique we obtained paths traversed by subjects and their appearance frequency in the cluster. Below figure $5\{\mathrm{a}, \mathrm{b}$ and $\mathrm{c}\}$ illustrate the paths whose frequencies are at least $\% 15$ in cluster one, two and three respectively, with their percentage in the overall set. In terms of performance indicators, cluster1 includes the subjects that have the best overall performance whereas the cluster3 includes the subjects that demonstrated the worst overall performance.

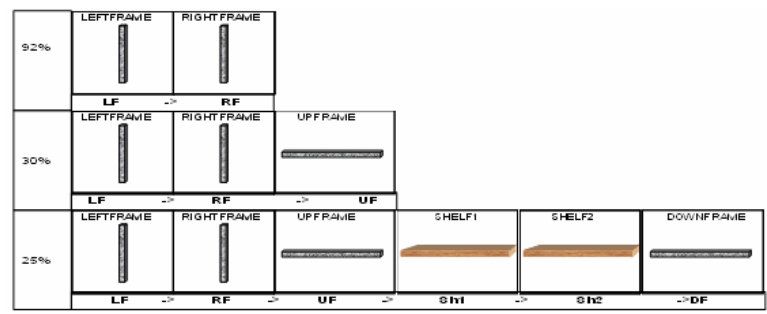

Figure 5-a: Paths from cluster I 


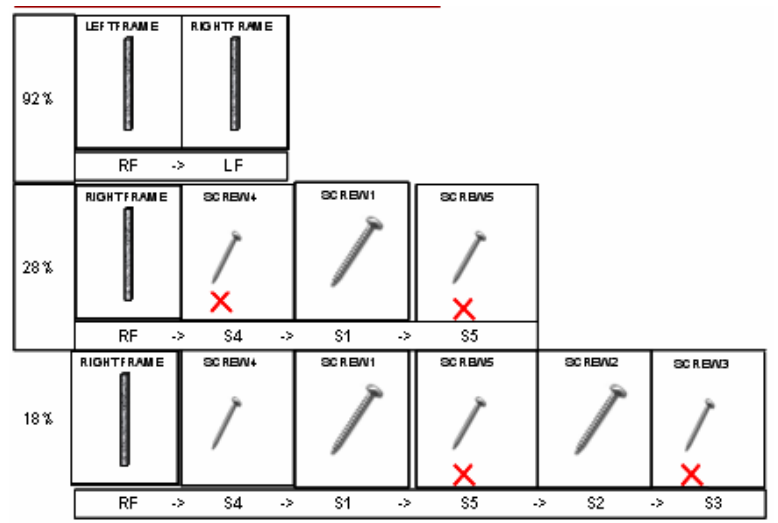

Figure 5-b: Paths from cluster II

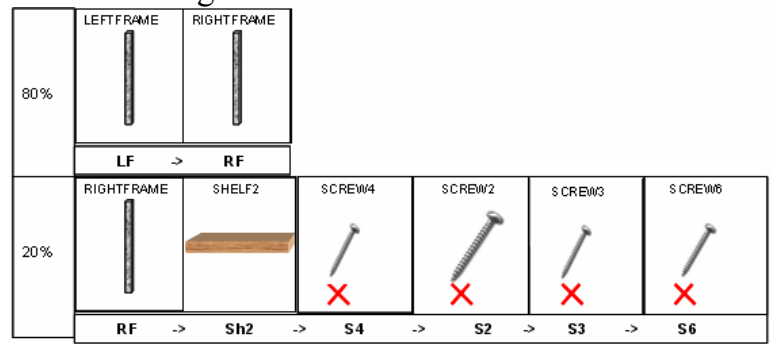

Figure 5-c: Paths from cluster III

Figure 5: Results from the clustering algorithm. Screws and large items found as the problematic parts in the assembly

As result we found violating sub paths that repeated in each cluster. Number of violating paths is 57 in the experiment, which means 4.75 tasks per user. While the shortest observed pattern consists of one event, the longest observed pattern consists of 11 events. Mean path length is 3.14. However none of the long patterns were frequent. It is observed that frequently violating sub paths consist of only one or two events as illustrated in figure 6 .

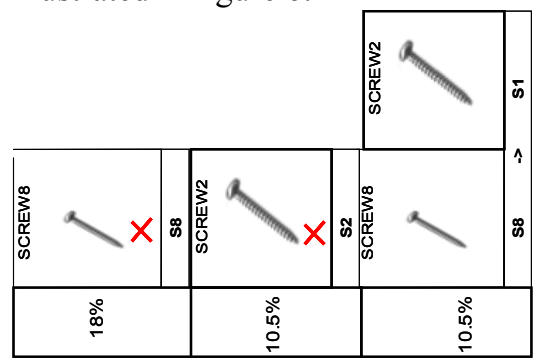

Figure 6. Violating sub paths: $X$ indicates the failure of a task

In the second test, where problems identified during the initial test are improved, we find a significant performance improvement. For example, distinguishing the different types of screws by coloring reduced the cancellations by $80 \%$. The significance in here is that the developed virtual reality based data accusation and analysis tool is capable of identifying problems in the design from the user point of view. Furthermore, improvements made in the design are verified quantitatively by the proposed model.

\section{Conclusions}

Today significant amount of man power and money are invested in designing and marketing of products or systems that serve to consumers' needs and expectations at the highest level. For this purpose we have introduced a VR based automated end-user evaluation methodology to provide automated support for decision makers. We integrate results from different fields to design a toolkit that enables automated analysis of behavioral data for all kinds of systems. The main idea of proposed analysis methodology is comparison of user paths with expected paths and with each other. To detect those paths in voluminous data we have implemented methods that can handle mining traversal patterns in sequential data. Once patterns are found we can compare the patterns with each other and process model. The final outcome of the proposed information processing technique gives insight about the users' way of dealing with certain tasks in a system or product. Moreover, the proposed method reveals the common paths followed by low-performance highperformance users. To validate the effectiveness of the proposed method, experimental study is conducted and results clearly demonstrate that the proposed methodology is capable of capturing user behavior from the virtual interaction with a system. Potential applications of this work are design process, training and after sales support like maintenance guidance, design for assembly and maintenance.

\section{References}

[1] Agrawal, R., Gunopulos, D., Leymann, F. (1997) Mining Process Models from Work Flow Logs. Research Report RJ 10100 (91916), IBM Almaden Research Center, San Jose California

[2] Agrawal, R., Srikant, R. (1995). Mining Sequential Patterns. In: Proc. of the Int'l Conference on Data Engineering (ICDE), Taipei, Taiwan, March 1995.

[3] Borges, J., A. (2000). Data Mining Model to Capture User Web Navigation Patterns. Unpublished doctoral dissertation, University College London, London

[4] Chen, M., Park, J. S., Yu, P. S. (1998) Efficient data mining for traversal patterns. IEEE Transactions on Knowledge and Data Engineering, 10(2):209--221, March/April 1998. 
[5] Cook, J. (1996) Process Discovery and Validation through Event-Data Analysis. Software Engineering Research Laboratory, Department of Computer Science, University of Colorado, Retrieved March 2, 2006, from http://citeseer.ist.psu.edu/87261.html

[6] Dunham, M. H., Xiao, Y. Efficient Mining of Traversal Patterns. (2001). In: Data \& Knowledge Engineering 39 191-214

[7] Hammontree, M., Weiller, P., Nayak, N. (1994). Remote Usability Testing. In: Interactions, July 1994, pp. 21-25

[8] Hilbert, D. M., Redmiles, D. F. (2000).Extracting Usability Information from User Interface Events. In: ACM Computing Surveys, Vol. 32, No. 4, December 2000, pp. 384-421.

[9] Ivory, Y. M., Hearst, M. A. (2001). The State of the Art in Automating Usability Evaluation of User Interfaces. In: ACM Computing Surveys, Vol. 33, No. 4

[10] Kuutti, K., Battarbee, K., Saade, S., Mattelmaki, T., Keinoen, T., S., Teirikko, T., Tornberg, A.(2001).Virtual prototypes in usability testing. In: Proceedings of the $34^{\text {th }}$ Hawaii International Conference on System Sciences-2001

[11] Oh, H., Yoon, S. (2004). What Virtual Reality Can Offer To Furniture Industry? In: Journal of Textile and Apparel, Technology and Management. Vol. 4, Issue 1, Summer 2004

[12] Paternò, F., Lecerof, A. (1998).Automatic Support for Usability Evaluation. In: IEEE Transactions on Software Engineering, Vol. 24, No. 10

[13] Sanderson, P.M., Scott, J.J.P., Johnston, T., Mainzer, J., Watanabe, L.M., James, J.M. (1994). MacSHAPA and the Enterprise of Exploratory Sequential Data Analysis (ESDA). In: International Journal of Human- Computer Studies, Vol. 41, 1994 